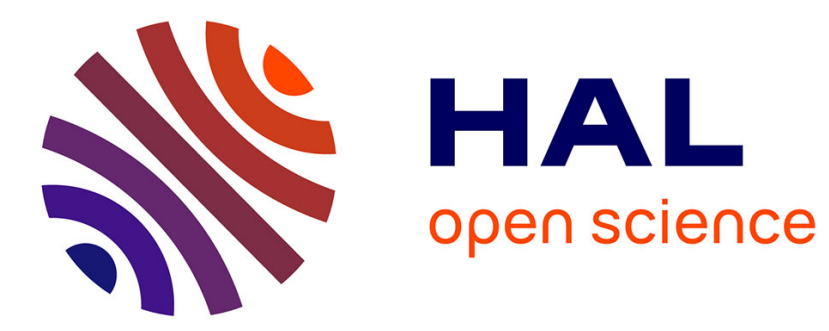

\title{
Prostate biopsies assisted by comanipulated probe-holder: first in man
}

Marie-Aude Vitrani, Michael Baumann, David Reversat, Guillaume Morel, Alexandre Moreau-Gaudry, Pierre Mozer

\section{- To cite this version:}

Marie-Aude Vitrani, Michael Baumann, David Reversat, Guillaume Morel, Alexandre Moreau-Gaudry, et al.. Prostate biopsies assisted by comanipulated probe-holder: first in man. International Journal of Computer Assisted Radiology and Surgery, 2016, 10.1007/s11548-016-1399-y · hal-01312297

\section{HAL Id: hal-01312297 \\ https://hal.science/hal-01312297}

Submitted on 5 May 2016

HAL is a multi-disciplinary open access archive for the deposit and dissemination of scientific research documents, whether they are published or not. The documents may come from teaching and research institutions in France or abroad, or from public or private research centers.
L'archive ouverte pluridisciplinaire HAL, est destinée au dépôt et à la diffusion de documents scientifiques de niveau recherche, publiés ou non, émanant des établissements d'enseignement et de recherche français ou étrangers, des laboratoires publics ou privés. 


\title{
Prostate biopsies assisted by comanipulated probe-holder: First in Man
}

\author{
Marie-Aude Vitrani · Michael Baumann . \\ David Reversat · Guillaume Morel · Alexandre \\ Moreau-Gaudry · Pierre Mozer
}

Received: date / Accepted: date

\begin{abstract}
Purpose: a comanipulator for assisting endorectal prostate biopsies is evaluated through a first-in man clinical trial. This lightweight system, based on conventional robotic components, possesses 6 degrees of freedom. It uses 3 electric motors and 3 brakes. It features a free mode, where its low friction and inertia allow for natural manipulation of the probe and a locked mode, exhibiting both a very low stiffness and a high steady state precision.

Methods: Clinical trials focusing on the free mode and the locked mode of the robot are presented. The objective is to evaluate the practical usability and performance of the robot during clinical procedures. A research protocol for a prospective randomized clinical trial has been designed. Its specific goal is to compare the accuracy of biopsies performed with and without the assistance of the comanipulator.

Results:The accuracy is compared between biopsies performed with and without the assistance of the comanipulator, across the 10 first patients included in the trial. Results show a statistically significant increase of the precision.
\end{abstract}

Keywords Robotics · Comanipulation · First-in-Man · prostate biopsy

M.-A. Vitrani, D. Reversat, G.Morel and P. Mozer are with (1) Sorbonne Universités UPMC Univ. Paris 06, UMR 7222, ISIR, Paris, France, (2) INSERM, U1150, Agathe-ISIR, F-75005, Paris France, (3) CNRS, UMR 7222, ISIR, F-75005, Paris, France. Email: \{reversat, mozer, vitrani, morel\}@isir.upmc.fr, $\cdot$ P. Mozer is with APHP Piti Salpêtrière Hospital, Urology Department, France, · M.Baumann is with Koelis Inc. Email: \{baumann\}@koelis . com, · A. Moreau-Gaudry is with (1) Laboratory of Techniques for Biomedical Engineering and Complexity Management Informatics, Mathematics and Applications, University Grenoble Alpes, Grenoble, France, (2) Laboratory of Techniques for biomedical engineering and complexity management informatics, mathematics and applications, National Center for Scientific Research, Grenoble, France, (3) Investigation Clinical Center 1406, National Institute of Health and Medical Research, Grenoble, France, (4) Technological Innovation - Investigation Clinical Center, Department of Public Health, University Hospital, Grenoble, France . Email: \{alexandre.moreau-gaudry\}@imag.fr. This work is partially funded french state funds managed by the ANR within the Investissements d'Avenir programme (Labex CAMI) under reference ANR-11-LABX-0004. 


\section{Introduction}

\subsection{Prostate cancer}

Prostate cancer $(\mathrm{PCa})$ is the fourth leading cause of cancer death in the world. With more than 900.000 new cases of prostate cancer each year in the world [1], PCa is one of the major public health problems, with growing incidence about 1 man in 7 that will be diagnosed with PCa during his lifetime, but whose therapeutic responses are not adapted to its growth. It is widely accepted today that a majority of patients would benefit in new clinical strategies as Active Surveillance or Focal Treatment.

The diagnosis is based on different exams. The Digital Rectal Evaluation and the Prostate Specific Antigen rate are performed during the screening phase while biopsy session is the only examination allowing to diagnose the cancer. It consists of sampling the prostate tissue using a biopsy needle. In 2013, more than 230.000 new prostate cancer cases have been detected in the USA, thanks to hundreds of thousands biopsy procedures [1].

The good distribution of biopsies in the prostate is the key aspect of the procedure as it limits the risk not to detect an existing tumor and the risk to overestimate a tumor extend. During standard examination, the urologist has to construct a mental 3D volume of the prostate using the $2 \mathrm{D}$ cross section provided by the ultrasound image. This is rather challenging, due to the mobility and deformations experienced by the prostate when moving the probe or inserting the needle, or caused by the patient motions [14,24].

A major technical difficulty arises from the desired precision for the needle placement, which is typically a few $\mathrm{mm}[15,23]$.

\subsection{Robotic devices to assist for prostate needle insertion}

Precisely positioning needles in a prostate is of crucial importance in terms of public health. It is required both for prostate biopsies and for brachytherapy, which consists of inserting radioactive seeds through a needle across the prostate volume in order to irradiate the cancerous tissue. Robotic assistance to needle placement in the prostate has been the object of interest for the robotics community in the past years. A recent exhaustive overview of these systems can be found in [12]. Two criteria can be used to categorize these systems:

- Needle insertion path: transrectal or transperineal

- Images used for guidance: ultrasonic images or MRI

Transperineal needle insertion ( $[5,7,9,10,12,17,18,27])$ involves, most of the time, a general anesthesia, which means more important risks and higher cost. It is mainly devoted to brachytherpy and not to biopsies, apart from [22], where a transperineal access is proposed for biopsies.

Using an MRI, as proposed in [11,13,16,22], induces a cost increase which limits its adoption, in particular for biopsy procedures whose cost should be limited as it is a common diagnostic procedure.

To avoid limitation form both MRI imaging and transperineal access, our system uses ultrasound images and allows transrectal needle insertion, as proposed in [13,21]. 
1.3 Assistance for prostate needle insertion through comanipulation

A major difference of our approach with respect to all the above-cited systems lies in the fact that the robotic device, called Apollo, does not move autonomously. Rather, it is comanipulated.

The urologist and the robot both manipulate the probe. Thus, with Apollo, the probe positioning is left to the urologist. During the positioning phase, the robot, whose end-effector is attached to the probe, shall leave the probe motion as free as possible. This first function is called the free mode. Once the probe has been manually positioned, Apollo provides a second function: the locked mode, during which the urologist has his/her hands free to perform the needle placement and the biopsy. Note that Apollo offers other advanced assistance functions, such as prostate-probe contact force enhancement [26], or autonomous fine positioning based on 3D USI [20]. These functions were not used in the present clinical study.

Being used as a simple free-locked device in the present clinical study, Apollo is comparable with the system Artemis commercialized by Eigen [2]. Two major differences between Apollo and Artemis can be noticed.

First, Artemis is to be registered to the patient as its Remote Center of Motion (RCM, which is a fixed point through which the transducer axis is constrained to pass) needs to precisely coincide with patient's anus. This induces a specific and careful procedure for the arm installation. On the contrary, Apollo possesses 6 degrees of freedom which allows for rough positioning of the base, simply accounting for workspace (reach) constraints. Apollo's kinematics is detailed in Sec. 2.1 .

Second, Artemis is not active. In other words, whenever the urologist locks the device with brakes and releases the transducer, one may observe a probe displacement due to the fact that the force balance has been changed. Only a very high stiffness of the arm can limit this deformation. Because the patient is awake and may move, this rigidity is not desirable. On the contrary, when set in locked mode, Apollo exhibits a low stiffness. Meanwhile, thanks to its actuators and its controller (see Sec. 2.3), it can reach with a high precision the locked position in spite of disturbances.

We hypothesize that this last characteristics is of high impact on the clinical practice as it improves precision. However, to our knowledge, there is no evaluation of the probe displacement when the locked mode is turned on with Artemis. The first-in-man trial reported in Sec. 3 aims at comparing the biopsy accuracy when performed with and without Apollo's assistance. A clinical research protocol for a prospective randomized clinical trial has been designed to evaluate, for the first time, on patients, this non CE marked innovative medical device. A device called Urostation Touch ${ }^{\circledR}$ provides ground thruth measurements. Results for the ten first patients (five with robot and five without) included in this study show an increase of the precision thanks to the robot.

\section{Proposed system}

In this section, the main characteristics of Apollo are presented. A more detailed description, together with an in vitro evaluation can be found in [19]. 


\subsection{Apollo's kinematics}

As evidenced from clinical data in [25], the required workspace for the probe main axis can be modeled by a cone, whose origin coincides with the anatomical entry point, and the angle is typically 60 degrees, see Fig. 1, upper left corner. A picture of Apollo is given in Fig. 1.

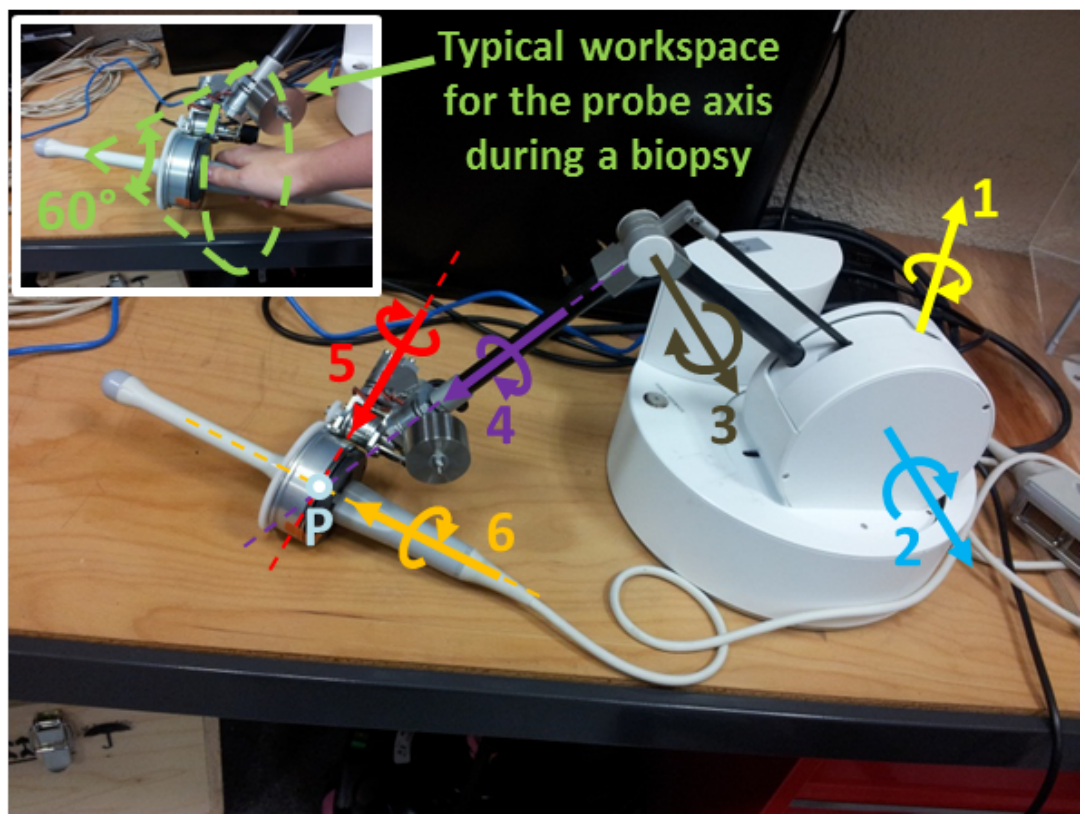

Fig. 1 General view of the proposed TRUS probe comanipulator called Apollo

Apollo is made of 6 pivot joints serially assembled according to a conventional anthropomorphic geometry: the 3 first joints form the shoulder and the elbow, while the wrist is composed of the three last joints, whose axes coincide at a point $P$, see Fig. 1 . The geometry is completely described by Table 1 according to Denavit and Hartenberg notation, [8].

\begin{tabular}{||c|c|c|c|c||}
\hline$i$ & $\alpha_{i-1}$ & $a_{i-1}$ & $d_{i}$ & $\theta_{i}$ \\
\hline 1 & 0 & 0 & 0 & $\theta_{1}$ \\
\hline 2 & $-\pi / 2$ & 0 & 0 & $\theta_{2}$ \\
\hline 3 & 0 & $0.25 \mathrm{~m}$ & 0 & $\theta_{3}$ \\
\hline 4 & $\pi / 2$ & 0 & $0.3 \mathrm{~m}$ & $\theta_{4}$ \\
\hline 5 & $-\pi / 4$ & 0 & 0 & $\theta_{5}$ \\
\hline 6 & $\pi / 2$ & 0 & 0 & $\theta_{6}$ \\
\hline
\end{tabular}

Table 1 Denavit and Hartenberg parameters of the comanipulator

The last pivot axis is designed in such a way that it leaves an $8 \mathrm{~cm}$ diameter cylindrical hole whose axis coincides with the rotational axis. An interface part is designed to adapt to any specific probe shape and to connect to the robot end-effector. This part is fixed on the probe and can be placed into the robot end effector thanks to a mechanical connector involving magnets. 


\subsection{Actuation}

In order to maintain a high transparency (low friction, low weight, low inertia) for the robot's free mode, while being able of maintaining the biopsy target at a precise location despite unknown disturbances in the locked mode, a hybrid actuation system is chosen:

- For the three wrist joints, small electromagnetic brakes are installed (Kebco 01.P1.300). The control of the brakes is binary: the brakes are either locked (ON), which corresponds to the unpowered state, or free (OFF), which corresponds to powered state.

- To be able to compensate for the possible displacements due to external forces, electric motors (Maxon RE35) are mounted on the three first joints.

Furthermore, to increase transparency in the free mode:

- the motors are placed near the robot base, in such a way that their mass does not significantly affect the robot's inertia.

- a cable transmission is used to limit joint friction.

- load springs are mounted on joints 2 and 3 to compensate for the robot weight.

The robot has been fabricated by Haption, a French company that commercializes high forces haptic interfaces [3].

\subsection{Assistance function}

When the urologist wants to move the probe, the robot, whose end-effector is attached to the probe, shall leave the probe motion as free as possible. This first function is called the free mode. On the contrary, once the probe has been positioned, the urologist can switch to the locked mode.

The robot links weight is balanced by counterweights and springs in such a way that there is no need for compensation of the robot weight by the actuators. However, to ease the comanipulation a gravity compensation is running to compensate for the weight of the probe.

During the free mode, brakes are OFF and nothing else but gravity compensation needs to be controlled as the robot was designed to ensure high transparency and reversability, see Fig. 2.

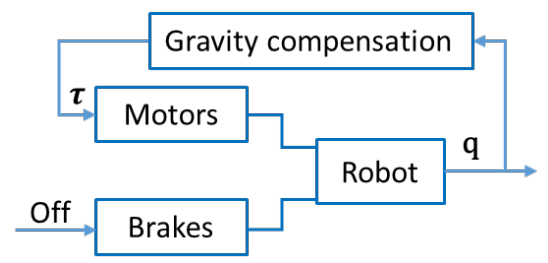

Fig. 2 Control loop to achieve Free mode

For the locked mode, a controller was developed to obtain simultaneously low stiffness and high precision. An inner loop compensates for gravity and emulates a spring between actual and desired locations. An external integrator is added to ensure precise positioning, see Fig. 3. This integrator is saturated to avoid windup. 
As demonstrated in [19], even though the inner loop spring exhibits a low stiffness, this system allows to compensate for displacements that occur when the urologist releases the probe, within a few seconds.

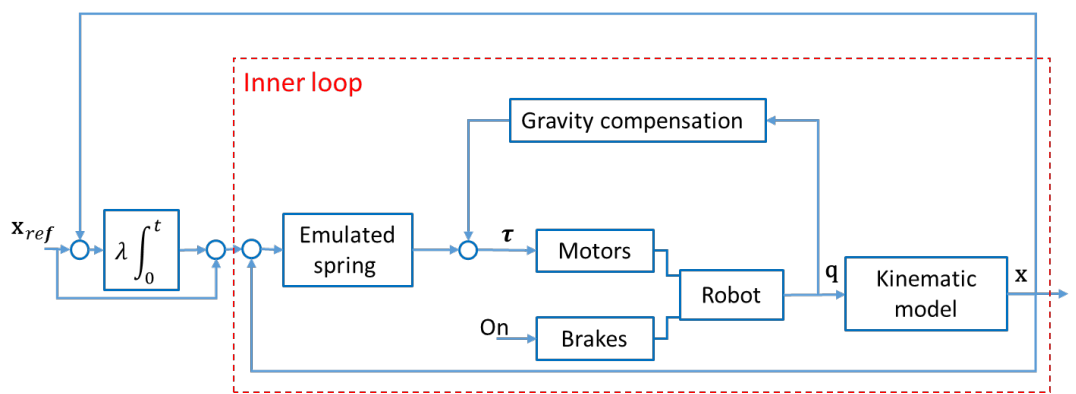

Fig. 3 Control loop to achieve Locked mode

\section{Clinical trial}

Following the successful in vitro and in cadavero experiments [19], a clinical trial focusing on the free mode and the locked mode of the robot was prepared. The objectives are to evaluate the practical usability and performance of the robot on patients, with a main objective focused on the accuracy of biopsies performed with and without the assistance of the medical device Apollo.

An exhaustive risk analysis was performed to identify and control potential risks for patients and users. Furthermore, the robotic system was improved to comply with the applicable norms for medical devices, in particular the norms for basic safety of medical electrical equipment (EN 60601-1) and for medical software lifecycle process (IEC 62304).

A clinical research protocol has been designed as a prospective randomized clinical trial, with respect to the scientific state-of-the-art and the current regulatory frame related to clinical evaluation with non CE marked devices. The sponsor is the Grenoble University Hospital and the clinical trial has been authorized by the relevant french regulatory bodies: Agence Nationale du Médicament et des Produits de Santé (ANSM), the ethical comitee (Comité de Protection des Personnes Sud-Est V, CPP) and the Commission Nationale de l'Informatique et des Libertés (CNIL). The authorization number is NCT02132975.

Twenty patients were included in this proof of concept study. The first patient (without robot) was included in November 2014, after obtaining the permissions to conduct the trial by all legal authorities, making it possible to perform the first intervention with robotic assistance in march 2015.

\subsection{Protocol}

\subsubsection{General installation}

Figure 4 shows the overall system installation in the operationing room. 


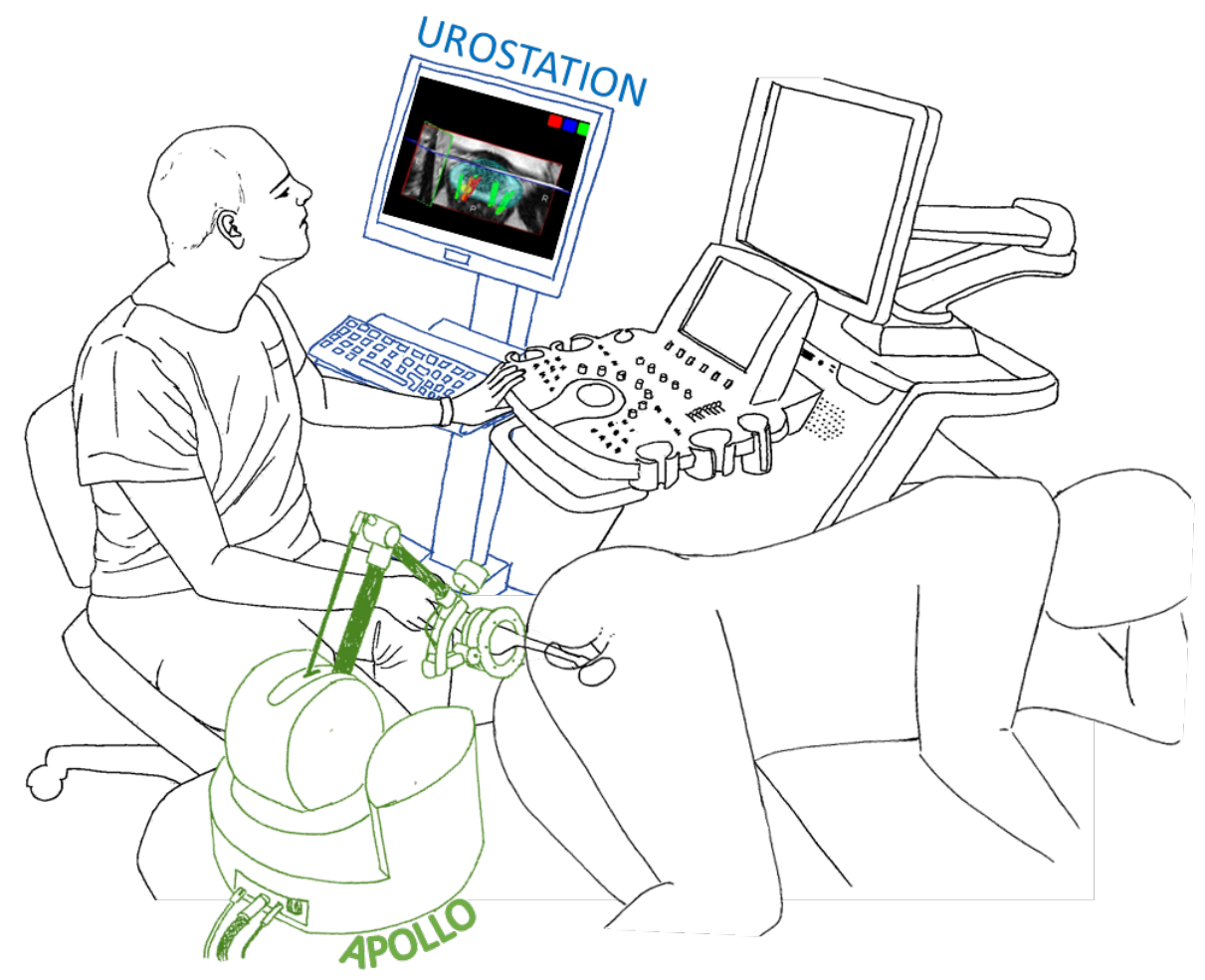

Fig. 4 Urostation Touch ${ }^{\circledR}$ and Apollo installation

In order to monitor the adequate positioning of the probe, the urologist was using a Urostation Touch ${ }^{\circledR}$, produced by the company Koelis (La Tronche, France), [4]. This system, which is approved for clinical use, is connected to a 3D ultrasound machine (Samsung V20). It is a mobile platform integrating several applications to assist prostate biopsy procedures with 3D imaging and intraoperative image fusion capabilities, within the usual clinical routine.

Urostation Touch ${ }^{\circledR}$ connects to an ultrasound scanner equipped with a 3D endocavitary probe. It receives, stores and processes 3D Dicom images of the prostate as the physician performs the biopsy procedure. It combines full-3D TRUS and automatic organ-based tracking [6] to create and visualize in real time the 3D mapping of biopsy samples, see Fig. 5.

Furthermore, it offers to the urologist the possibility to perform what is called a "virtual biopsy": the urologist records a 3D US image which is registered to the reference 3D image. Knowing the displacement computed by the registration algorithm, the Urostation Touch ${ }^{\mathrm{R}}$ displays the expected location of the biopsy needle. 


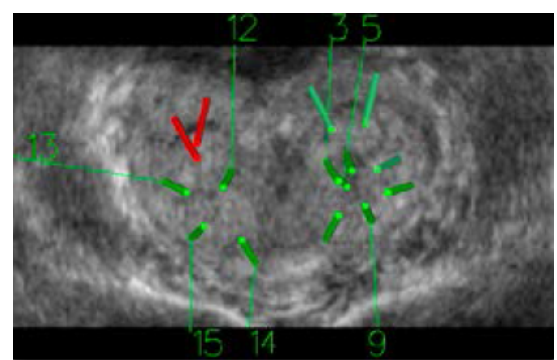

Fig. 5 3D mapping of biopsy samples from Urostation Touch ${ }^{\circledR}$

\subsubsection{Scenario}

For the robotized procedures, the robot is installed in the operating room before the biopsy session starts, see Fig. 6.

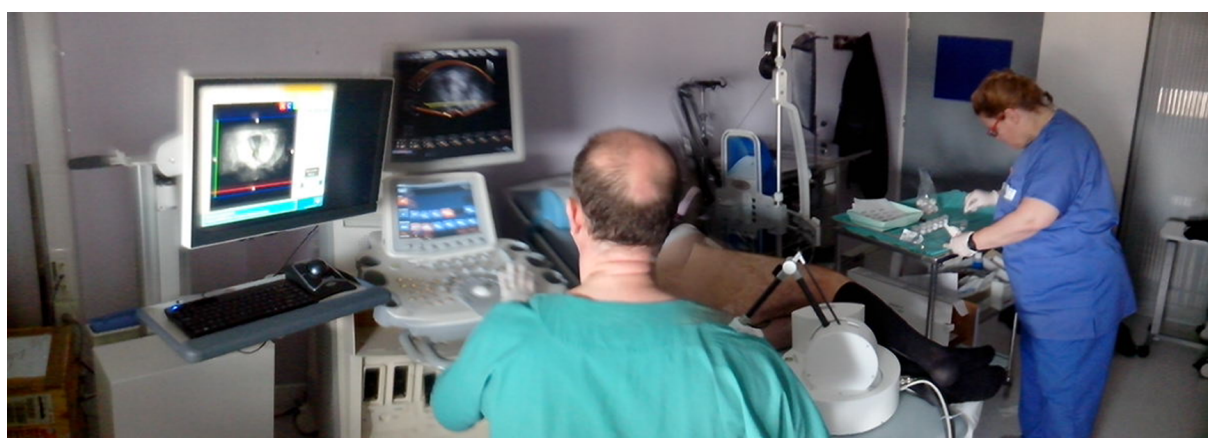

Fig. 6 Room installation with the robot Apollo and the Urostation Touch ${ }^{\circledR}$

The patient lies on left-lateral decubitus position. The robot (when used) is installed on patient's bed, behind his legs and the urologist connects the probe to the robot. An endorectal ultrasound probe fitted with a needle guide is inserted in the patient rectum Fig. 7-left. A local anesthesia is then preformed.

First, the probe is inserted into the patient's rectum (either manually or, for patients with robot, in the free mode). Immediately after introducing the probe, the urologist records a reference 3D US image. This reference image is displayed on a screen interface.

Then the urologist moves the probe toward a first desired biopsy site. To this aim, he/she uses the real time 2D US image and mental reconstruction of the anatomical geometry.

When the urologist thinks that the probe position is satisfactory for the biopsy, he/she makes a 3D US acquisition to visualize the "virtual biopsy". When the virtual biopsy is satisfactory (i.e. the currently targeted site as displayed in the 3D reference image is adequate), the urologist performs the biopsy and finally acquires a new 3D US image. In other words, for each biopsy, both the intended location and the effective location are recorded.

When done, the urologist can proceed to the next biopsy and repeat the above procedure until all the biopsies have been done. During the trial, the urologist had to position the probe at twelve different locations, according to the conventional sextant scheme (see Fig. 7-up- 


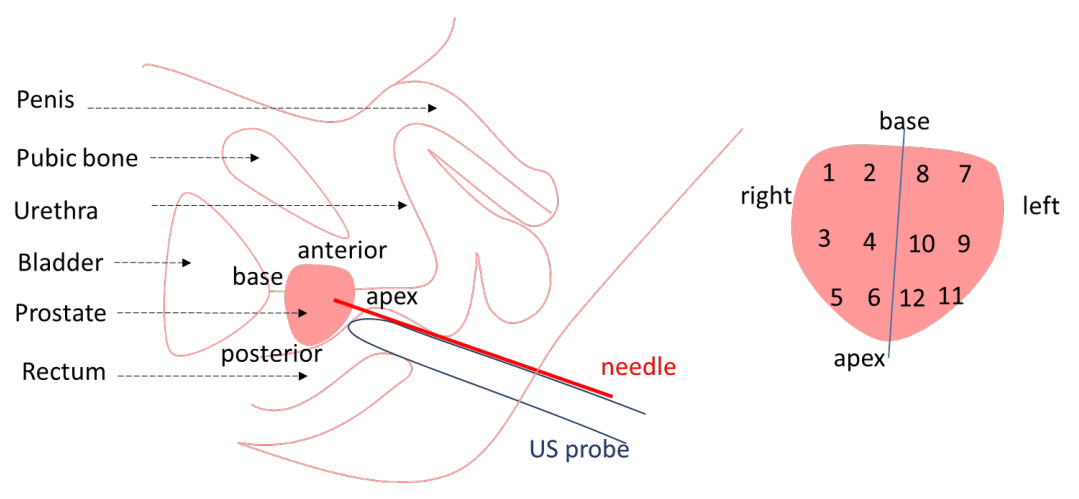

Fig. 7 Anatomical description and Sextant scheme

left). This scheme is a general guideline used by the urologist but is not converted to exact target locations visualized in the reference $3 \mathrm{D}$ images.

The urologist also had to perform 3 supplementary biopsies targeted toward one suspicious lesion observed in MRI data (presenting a suspicious lesion from a preoperative MRI examination was indeed a criterion for inclusion in the clinical trial). This lesion is made visible in the reference 3D image displayed on the screen, thanks to an MRI-3DUS registration algorithm implemented in the Urostation Touch ${ }^{\circledR}$.

For a given biopsy site, when the robot is not used, the urologist needs to manually maintain the probe from the beginning of the first 3D acquisition (virtual biopsy) the end of the second 3D acquisition (real biopsy). This corresponds to the current practice in clinical routine when using the Urostation Touch ${ }^{\circledR}$. On the contrary, when using the robot, the urologist selects the locked mode at the beginning of the first acquisition. He/she switches back to the free mode after the end of the second acquisition, when moving the probe toward the next biopsy location.

We hypothesize that the robot can minimize the displacement that occurs between the virtual biopsy (intended location) and the real biopsy (effective location). This is what was measured during the trial.

\subsection{Results}

The overall clinical study included 20 patients and three different urologists with various levels of expertise. We present here a first statistical analysis performed for the 10 first patients. Among them, two patients (patients 1 with robot and 8 without robot) were discharged from the study: the first one because of a needle guide material problem, the second one because he did not stop his anticoagulant treatment. The following data are thus given for 8 patients ( 4 with robot and 4 without robot). Patient clinical characteristics are given in Table 2.

Hereafter, the quantitative parameters are described using mediane, $25^{\text {th }}$ and $75^{\text {th }}$ percentil. The qualitative indicators are expressed as number and percentage. Due to the small number of patient, a non parametric Mann-Whitney-Wilcoxon test has been chosen. 
Table 2 Patients clinical characteristics.

\begin{tabular}{lll}
\hline Characteristics & with Robot $(n=4)$ & without robot $(n=4)$ \\
\hline Age (year) (NA= 0), median [IQ] & $60[58.5 ; 62.2]$ & $71[63 ; 75.5]$ \\
BMI (NA=0), median [IQ] & $25.8[24.9 ; 26.2]$ & $28.4[26.4 ; 30.8]$ \\
PSA rate (ng/ml) (NA=0), median [IQ] & $11.1[6.3 ; 15.8]$ & $9.3[8.2 ; 10.3]$ \\
Prostate Volume (cm3) (NA=0), median [IQ] & $39.5[28.5 ; 54.2]$ & $56.5[50 ; 71.5]$ \\
Suspected lesion size (mm) (NA=0), median [IQ] & $7.5[6.8 ; 8.5]$ & $14[11 ; 19.5]$ \\
\hline
\end{tabular}

\subsubsection{Main criterion : distance between virtual and real biopsies}

The main criterion analysed is the distance between the virtual (desired) biopsy and the real one. A typical 3D map for the biopsies, as computed by the Urostation Touch ${ }^{\mathrm{R}}$ for a given patient, is showed on Fig. 8

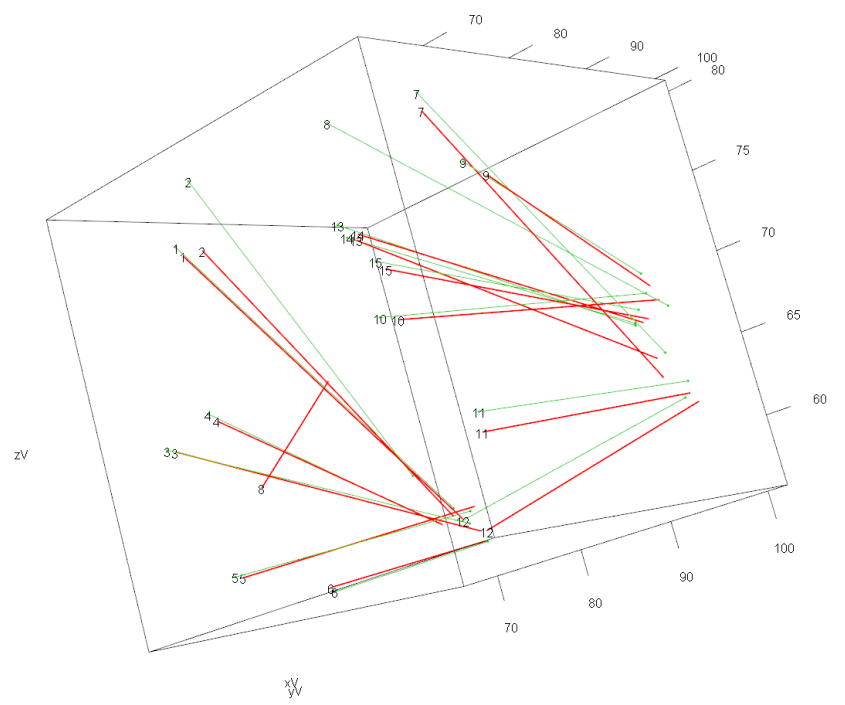

Fig. 8 3D maps of the biopsies for patient 4 with robot ; in red virtual biopsie - in green real biopsies

The results are given for the 8 patients in Table 3 .

The statistics for the median distance per patient (across fifteen biopsies) are given in Table 4. The median distance between virtual and real biopsies per patient is lower thanks to the robot $(-0.77 \mathrm{~mm}=-46 \%)$ with a statistical significance $(p=0.029<0.05)$.

\subsubsection{Secondary criteria}

Several other criteria were defined in order to evaluate the impact of the use of the robot on the clinical practice.

The complete duration of the procedure (from the beginning of the installation to the patient's exit) was 33 minutes without robot and 41 minutes with the robot (median values 
Table 3 distance between virtual and real biopsies (in $\mathrm{mm}$ ).

\begin{tabular}{llllllllll}
\hline Patient & Group & Min & Q1 & Median & Mean & Q3 & Max & STD & N \\
\hline 2 & manual & 0.88 & 1.55 & 2.08 & 2.39 & 3.03 & 5.03 & 1.2 & 15 \\
3 & manual & 0.73 & 1.51 & 1.78 & 1.97 & 2.52 & 4.3 & 0.91 & 15 \\
4 & robot & 0.27 & 0.45 & 0.74 & 0.93 & 1.17 & 2.34 & 0.63 & 14 \\
5 & manual & 1.07 & 1.33 & 1.56 & 1.82 & 2.01 & 3.16 & 0.65 & 15 \\
6 & robot & 0.33 & 0.52 & 1.01 & 1.28 & 1.74 & 3.14 & 0.94 & 15 \\
7 & robot & 0.24 & 0.44 & 1.07 & 1.08 & 1.52 & 2.8 & 0.75 & 15 \\
9 & manual & 0.65 & 0.91 & 1.27 & 1.34 & 1.65 & 2.28 & 0.51 & 15 \\
10 & robot & 0.16 & 0.54 & 0.8 & 0.9 & 1.2 & 1.66 & 0.45 & 14 \\
\hline
\end{tabular}

Table 4 Median distance between virtual and real biopsies (in $\mathrm{mm}$ ) per group.

\begin{tabular}{lllllllll}
\hline Group & Min & Q1 & Median & Mean & Q3 & Max & STD & N \\
\hline robot & 0.74 & 0.78 & 0.9 & 0.9 & 1.02 & 1.07 & 0.16 & 4 \\
manual & 1.27 & 1.49 & 1.67 & 1.67 & 1.86 & 2.08 & 0.34 & 4 \\
\hline
\end{tabular}

across the patients). This 8 minutes (25\%) increase was due to installation of the robot prior to the patient arrival in the room, as well as to the time spent to show the robot and its control modes to the patient. This difference is not statistically significant $(p=0.057>0.05)$ at this stage of the study.

Another secondary criterion was the possibility to explore the overall prostate with the robot. For all the 4 patients, it has been observed that the urologist was not disturbed by any workspace limitation of the robot.

Some qualitative results were also obtained through questionnaires to the urologist and to the patient after the procedure with robot. Urologists were asked about resistance felt during the free mode. They had to choose their answer between none, slight, moderate or high. The urologists declared feeling "no resistance" when using the robot in the free mode, except, for two sessions, for the rotation around the probe main axis (a moderate resistance was then reported).

The robot was "compact" to their opinion (they had to choose between "no presence noticeable", "compact", "moderately" or "highly cumbersome").

Urologists judged "very useful" the locked mode (they had to choose between useless, comfortable, useful or very useful).

Three patients said they were reassured thanks to the robot use, the fourth one declared it did not change his apprehension. They felt no differences or forces due to switch between free and locked mode.

Finally, only one complication occurred during these experiments: a patient presents a urinary retention. It was not due to the robot. For one patient, the robot reached the security force saturation level to maintain the probe but this did not impact the clinical procedure as this level was deliberately set far below the real capacity of the robot.

\subsection{Discussion}

Through these experiments, we were able to validate the overall set-up and procedure. We did not encounter any difficulty to perform the biopsies with the robot even with the rough positioning of the robot with respect to the patient. 
Obviously this study is a preliminary clinical validation. One cannot state about the clinical added value based on this experiment. To validate the assistance provided by the robot and its overall set-up (workspace, forces of the robot) a larger protocol should be defined with more surgeons and more patients. Furthermore, several patient characteristics (age, prostate volume, suspected lesion size) seem to be significantly different between the two groups despite the randomized assignment. The effect of these difference were not evaluate because of the small number of patients included in the study. This should be investigated through a clinical study with more patients.

\section{Conclusion}

In this paper, we presented Apollo, a comanipulator for assisting endorectal prostate biopsies. This lightweight system, based on conventional robotic components, possesses 6 degrees of freedom. It features a free mode, where its low friction and inertia allows for natural manipulation of the probe and a locked mode, exhibiting both a very low stiffness and a high steady state precision.

A first evaluation with 10 human patients has been reported. Its main objective was to compare the accuracy of biopsies performed with and without the assistance of the medical device Apollo. Results exhibit a statistically significant increase of the precision.

Conflict of interest : The authors declare that they have no conflict of interest.

Research involving human participants: The research protocol for a clinical trial was authorized by the sponsor of the clinical study (Grenoble University Hospital) and the relevant legal authorities through the reference PROSBOT-Apollo Biopsies assisted by comanipulated robot - Pilot Clinical trial; 2015; Acceptance number NCT02132975.

This article does not contain any studies with animals performed by any of the authors.

Informed consent: Informed consent was obtained from all individual participants included in the study.

\section{References}

1. American cancer society. URL http://www.cancer.org

2. Eigen. URL www.eigen.com

3. Haption. URL http://www.haption.fr

4. Koelis. URL http://www.koelis.fr

5. Bassan, H., Hayes, T., Patel, R., Moallem, M.: A novel manipulator for $3 \mathrm{~d}$ ultrasound guided percutaneous needle insertion. In: Robotics and Automation, 2007 IEEE International Conference on, pp. 617-622 (April 2007)

6. Baumann, M., Mozer, P., Daanen, V., Troccaz, J.: Prostate biopsy tracking with deformation estimation. Medical Image Analysis 16(3), 562 - 576 (2012)

7. van den Bosch, M.R., Moman, M.R., van Vulpen, M., Battermann, J.J., Duiveman, E., van Schelven, L.J., de Leeuw, H., Lagendijk, J.J.W., Moerland, M.A.: Mri-guided robotic system for transperineal prostate interventions: proof of principle. Physics in Medicine and Biology 55(5), N133 (2010)

8. Denavit, J., Hartenberg, R.S.: A kinematic notation for lower-pair mechanisms based on matrices. Trans ASME J. Appl. Mech 23, 215-221 (1955)

9. Fichtinger, G., DeWeese, T.L., Patriciu, A., Tanacs, A., Mazilu, D., Anderson, J.H., Masamune, K., Taylor, R.H., Stoianovici, D.: System for robotically assisted prostate biopsy and therapy with intraoperative ct guidance. Academic Radiology 9(1), 60 - 74 (2002) 
10. Fichtinger, G., Fiene, J.P., Kennedy, C.W., Kronreif, G., Iordachita, I., Song, D.Y., Burdette, E.C., Kazanzides, P.: Robotic assistance for ultrasound-guided prostate brachytherapy. Medical Image Analysis 12(5), 535 - 545 (2008). Special issue on the 10th international conference on medical imaging and computer assisted intervention - MICCAI 2007

11. Fischer, G., Iordachita, I., Csoma, C., Tokuda, J., DiMaio, S., Tempany, C., Hata, N., Fichtinger, G.: Mricompatible pneumatic robot for transperineal prostate needle placement. Mechatronics, IEEE/ASME Transactions on 13(3), 295-305 (June 2008)

12. Hungr, N., Baumann, M., Long, J., Troccaz, J.: A 3-d ultrasound robotic prostate brachytherapy system with prostate motion tracking. IEEE Transactions on Robotics 28(6), 1382-1397 (2012)

13. Krieger, A., Iordachita, I., Guion, P., Singh, A., Kaushal, A., Menard, C., Pinto, P., Camphausen, K., Fichtinger, G., Whitcomb, L.: An mri-compatible robotic system with hybrid tracking for mri-guided prostate intervention. Biomedical Engineering, IEEE Transactions on 58(11), 3049-3060 (Nov. 2011)

14. Lagerburg, V., Moerland, M.A., Lagendijk, J.J., Battermann, J.J.: Measurement of prostate rotation during insertion of needles for brachytherapy. Radiotherapy and Oncology 77(3), 318 - 323 (2005)

15. Lu-Yao, G., Albertsen, P., Moore, D., Shih, W., Lin, Y., Di Paola, R., Barry, M., Zietman, A., O'Leary, M., Walker-Corkery, E., Yao, S.: Outcomes of localized prostate cancer following conservative management. JAMA 302(11), 1202-1209 (2009)

16. Patriciu, A., Petrisor, D., Muntener, M., Mazilu, D., Schar, M., Stoianovici, D.: Automatic brachytherapy seed placement under mri guidance. Biomedical Engineering, IEEE Transactions on 54(8), 1499-1506 (Aug.2007)

17. Phee, L., Xiao, D., Yuen, J., Chan, C.F., Ho, H., Choon, H., Christopher, C., Wan, S.N.: Ultrasound guided robotic system for transperineal biopsy of the prostate. In: Robotics and Automation, 2005. ICRA 2005. Proceedings of the 2005 IEEE International Conference on, pp. 1315-1320 (April)

18. Podder, T., Ng, W.S., Yu, Y.: Multi-channel robotic system for prostate brachytherapy. In: Engineering in Medicine and Biology Society, 2007. EMBS 2007. 29th Annual International Conference of the IEEE, pp. 1233-1236 (Aug.2007)

19. Poquet, C., Mozer, P., Vitrani, M.A., Morel, G.: An endorectal ultrasound probe comanipulator with hybrid actuation combining brakes and motors. Mechatronics, IEEE/ASME Transactions on 20(1), 186196 (2015). DOI 10.1109/TMECH.2014.2314859

20. Poquet Torterotot, C., Vitrani, M.A., Mozer, P., Morel, G.: Achieving high precision in prostate biopsy thanks to robot closed loop control based on 3d ultrasound imaging. In: Surgetica 2014, pp. 1-4 (2014)

21. Schneider, C., Okamura, A., Fichtinger, G.: A robotic system for transrectal needle insertion into the prostate with integrated ultrasound. In: Robotics and Automation, 2004. Proceedings. ICRA '04. 2004 IEEE International Conference on, vol. 1, pp. 365-370 Vol.1 (April-1 May)

22. Song, S.E., Cho, N., Fischer, G., Hata, N., Tempany, C., Fichtinger, G., Iordachita, I.: Development of a pneumatic robot for mri-guided transperineal prostate biopsy and brachytherapy: New approaches. In: Robotics and Automation (ICRA), 2010 IEEE International Conference on, pp. 2580-2585 (May 2010)

23. Stattin, P., Holmberg, E., Johansson, J.E., Holmberg, L., Adolfsson, J., Hugosson, J., on behalf of the National Prostate Cancer Register (NPCR) of Sweden: Outcomes in localized prostate cancer: National prostate cancer register of sweden follow-up study. Journal of the National Cancer Institute 102(13), 950-958 (2010)

24. Stone, N.N., Roy, J., Hong, S., Lo, Y.C., Stock, R.G.: Prostate gland motion and deformation caused by needle placement during brachytherapy. Brachytherapy 1(3), 154 - 160 (2002)

25. Torterotot, C., Mozer, P., Baumann, M., Vitrani, M., Morel, G.: Analysis of endorectal probe kinematics during prostate biopsies. In: Proc. of the Hamlyn Symposium on Medical Robotics, ISBN: 978-09563776-1-6, pp. 47-48 (2010)

26. Torterotot-Poquet, C., Vitrani, M.A., Mozer, P., Morel, G.: Ultrasound image-based comanipulation for enhanced perception of the contacts with a distal soft organ. In: Robotics and Biomimetics (ROBIO), 2011 IEEE International Conference on, pp. 1140-1146 (Dec.2011)

27. Wei, Z., Ding, M., Downey, D., Fenster, A.: 3d trus guided robot assisted prostate brachytherapy. In: J. Duncan, G. Gerig (eds.) Medical Image Computing and Computer-Assisted Intervention MICCAI 2005, Lecture Notes in Computer Science, vol. 3750, pp. 17-24. Springer Berlin Heidelberg (2005) 